\title{
BMJ Open Efficacy and safety of omalizumab in chronic rhinosinusitis with nasal polyps: a systematic review and meta- analysis of randomised controlled trials
}

\author{
Qingwu Wu ${ }^{1,2}$ Lianxiong Yuan, ${ }^{3}$ Huijun Qiu, ${ }^{1}$ Xinyue Wang, ${ }^{1}$ Xuekun Huang, ${ }^{1}$ \\ Rui Zheng, ${ }^{1,2}$ Qintai Yang (D) ${ }^{1,2}$
}

To cite: Wu Q, Yuan L, Qiu H, et al. Efficacy and safety of omalizumab in chronic rhinosinusitis with nasal polyps: a systematic review and meta-analysis of randomised controlled trials. BMJ Open 2021;11:e047344. doi:10.1136/ bmjopen-2020-047344

\section{- Prepublication history and} additional supplemental material for this paper are available online. To view these files, please visit the journal online (http://dx.doi.org/10.1136/ bmjopen-2020-047344).

$\mathrm{QW}, \mathrm{LY}$ and $H Q$ contributed equally.

Received 25 November 2020 Accepted 03 August 2021

Check for updates

(C) Author(s) (or their employer(s)) 2021. Re-use permitted under CC BY-NC. No commercial re-use. See rights and permissions. Published by BMJ.

For numbered affiliations see end of article.

\section{Correspondence to}

Dr Qintai Yang;

yangqint@mail.sysu.edu.cn and

Dr Rui Zheng;

zhengr8@mail.sysu.edu.cn

\section{ABSTRACT}

Objectives To assess the efficacy and safety of omalizumab for chronic rhinosinusitis with nasal polyps (CRSwNP) and to identify evidence gaps that will guide future research on omalizumab for CRSwNP.

Design Systematic review and meta-analysis.

Data sources A comprehensive search was performed in PubMed, Embase, Web of Science and the Cochrane Library on 13 October 2020.

Eligibility criteria Randomised controlled trials (RCTs) comparing omalizumab with placebo, given for at least 16 weeks in adult patients with CRSwNP.

Data extraction and synthesis Two independent authors screened search results, extracted data and assessed studies using the Cochrane risk of bias tool. Data were pooled using the inverse-variance method and expressed as mean differences (MDs) with 95\% Cls. Heterogeneity was assessed by the $\chi^{2}$ test and the $\mathrm{I}^{2}$ statistic.

Results A total of four RCTs involving 303 participants were identified. When comparing omalizumab to placebo, there was a significant difference in Nasal Polyps Score (MD $=-1.20 ; 95 \% \mathrm{Cl}$ -1.48 to -0.92$)$, Nasal Congestion Score (MD $=-0.67$; $95 \% \mathrm{Cl}-0.86$ to -0.48$)$, Sino-Nasal Outcome Test-22 $(\mathrm{MD}=-15.62 ; 95 \% \mathrm{Cl}-19.79$ to -11.45$)$, Total Nasal Symptom Score (MD=-1.84; 95\% Cl -2.43 to -1.25 ) and reduced need for surgery (risk ratio $(\mathrm{RR})=5.61$; $95 \% \mathrm{Cl} 1.99$ to 15.81). Furthermore, there was no difference in the risk of serious adverse events ( $(\mathrm{RR}=1.40 ; 95 \% \mathrm{Cl} 0.29$ to 6.80$)$, adverse events $(\mathrm{RR}=0.83 ; 95 \% \mathrm{Cl} 0.60$ to 1.15$)$ and rescue systemic corticosteroid (RR=0.52; $95 \% \mathrm{Cl} 0.17$ to 1.61 ).

Conclusions This was the first meta-analysis that identified omalizumab significantly improved endoscopic, clinical and patient-reported outcomes in adults with moderate to severe CRSwNP and it was safe and well tolerated.

PROSPERO registration number CRD42020207639.

\section{INTRODUCTION}

Chronic rhinosinusitis (CRS) is common and affects up to $5 \%-12 \%$ of the general population. ${ }^{1}$ It is defined as inflammation of the nose and the paranasal sinuses

\section{STRENGTHS AND LIMITATIONS OF THIS STUDY}

$\Rightarrow$ This systematic review and meta-analysis was based on a comprehensive search and included randomised controlled trials (RCTs).

$\Rightarrow$ Studies were low risk of bias, which was assessed by the Cochrane risk of bias tool.

$\Rightarrow$ Because the longest follow-up of four RCTs was only up to 26 weeks, there were too short to comprehensively and adequately assess the risks of side effect.

characterised by nasal congestion, nasal discharge, facial pressure and loss of smell. CRS with nasal polyps (CRSwNP) is a severe form of CRS and accounts for $18 \%$ of patients with CRS. ${ }^{2}$ CRSwNP is associated with adult-onset asthma, decreased health-related quality of life (HRQoL) $)^{34}$ and substantial economic burden. ${ }^{5}$ Many patients with CRSwNP often fail to achieve sufficient benefit from intranasal corticosteroids (INCS) or systemic corticosteroids (SCS) and/or functional endoscopic sinus surgery (FESS). ${ }^{6}$ Although FESS may be successful initially, relapse occurs in $20 \%$ of patients after 12 months, ${ }^{7}$ in $40 \%$ after 18 months ${ }^{8}$ and in $80 \%$ after 12 years despite ongoing INCS therapy. ${ }^{9}$ Therefore, novel treatments such as biologics are needed for CRSwNP.

Omalizumab (anti-IgE antibody) is one of the biologics and may help patients with severe CRSwNP. It was reported that omalizumab made their symptom better and shrank their polyps in small-size randomised controlled trials (RCTs). ${ }^{10} 11$ But some of its effectiveness and safety are not well known. Thus, some systematic reviews were conducted to assess the effectiveness and safety of it. But they found very little information or insufficient evidence about the use of omalizumab 


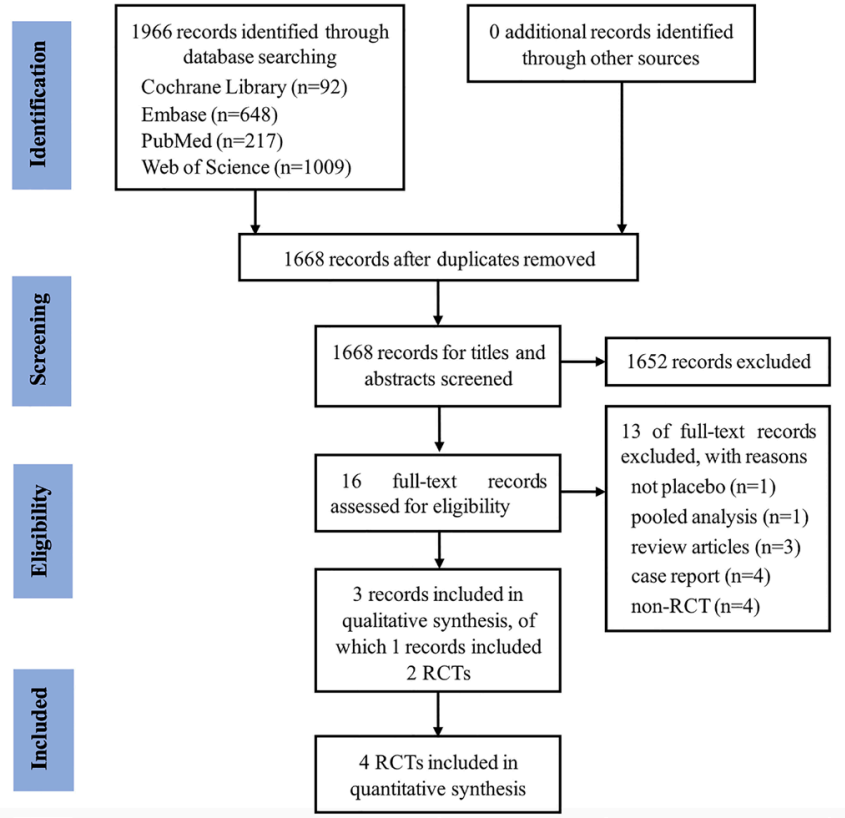

Figure 1 PRISMA flow diagram of the literature search. PRISMA, Preferred Reporting Items for Systematic Reviews and Meta-Analyses; RCTs, randomised controlled trials.

and cannot determine whether it was effective or not. ${ }^{12}{ }^{13}$ Currently, some well-designed RCTs about omalizumab for CRSwNP were published, ${ }^{14}$ which may provide us with some evidence. Therefore, this systematic review was conducted to evaluate the efficacy and safety of omalizumab vs placebo in adult patients with CRSwNP, and identify evidence gaps that will guide future research on omalizumab for CRSwNP.

\section{METHODS}

We performed a systematic review based on a priori protocol that was registered with PROSPERO (No. CRD42020207639). ${ }^{15}$ This review was reported according to the Preferred Reporting Items for Systematic Reviews and Meta-Analyses (PRISMA) statement ${ }^{16}$ (online supplemental additional file 1).

\section{Eligibility criteria}

(1) Population: adult patients (>18) with CRSwNP; (2) Intervention and comparison: studies comparing omalizumab with placebo, given for at least 16 weeks; (3) Primary outcomes: Nasal Polyps Score (NPS), Nasal Congestion Score (NCS) and Sino-Nasal Outcome Test-22 score (SNOT-22); Secondary outcomes: Total Nasal Symptom Score (TNSS), serious adverse events (SAEs), AEs, rescue SCS (RSCS) and reduced need for surgery (RNS); (4) Study design: RCTs and (5) Studies written and published in the English language were included.

\section{Search strategy and selection process}

A comprehensive search was performed in PubMed, Embase, Web of Science and the Cochrane Library on 13 October 2020. We used the following combined text and MeSH terms: 'nasal polyps', 'sinusitis' and 'omalizumab'. Search strategies for major databases are provided in online supplemental appendix 1 .

Titles and abstracts of the retrieved articles were then screened for their potential relevance by two reviewers (QW and LY). The full-text articles were obtained and assessed by the same reviewers to determine whether they met the inclusion criteria for this review. We resolved any differences by a discussion with a third author $(\mathrm{QY})$.

\section{Data extraction}

Two reviewers (HQ and XW) read full-text articles and extracted data using a predefined extraction form. Data were extracted on the following: first author, year of publication, patient characteristics, study methods and outcome data.

\section{Assessment of risk of bias}

In this review, the original version of the Cochrane 'Risk of bias' tool was used to assess the risk of bias in included studies. The risk of bias was assessed as 'low', 'high' or 'unclear' for each of the following six domains: sequence generation; allocation concealment; blinding of participants, personnel and outcome assessment; incomplete outcome data; selective reporting; other sources of bias (if required).

\section{Statistical analysis}

Study characteristics were shown in tables and described narratively. For dichotomous data, we planned to analyse treatment differences as a risk ratio (RR) calculated using the Mantel-Haenszel methods. For continuous outcomes, a generic inverse-variance method with fixed-effects models was used to calculate pooled mean differences (MD) and 95\% CI. Statistical heterogeneity was assessed by the $\chi^{2}$ test (with a significance level set at $\mathrm{p}<0.10)$ and the $\mathrm{I}^{2}$ statistic $\left(\mathrm{I}^{2} \geq 50 \%\right.$ indicates substantial heterogeneity). There are two large pharma-sponsored RCTs with most of the information and two smaller RCTs with effect sizes much larger and much smaller than the two main studies. A random-effects meta-analysis may exacerbate the effects of the bias and a fixed-effect analysis will be affected less, although strictly fixed-effect analysis will also be inappropriate. ${ }^{17}$ Therefore, we choose a fixed-effect analysis in this study. Sensitivity analysis were performed, which included the removal of each single study from the meta-analysis one at a time and recalculation of the summary effect. The possibility of publication bias was assessed by constructing a funnel plot if sufficient studies $(>10)$ were available for an outcome. All meta-analyses were conducted by the Review Manager (V.5.3). 


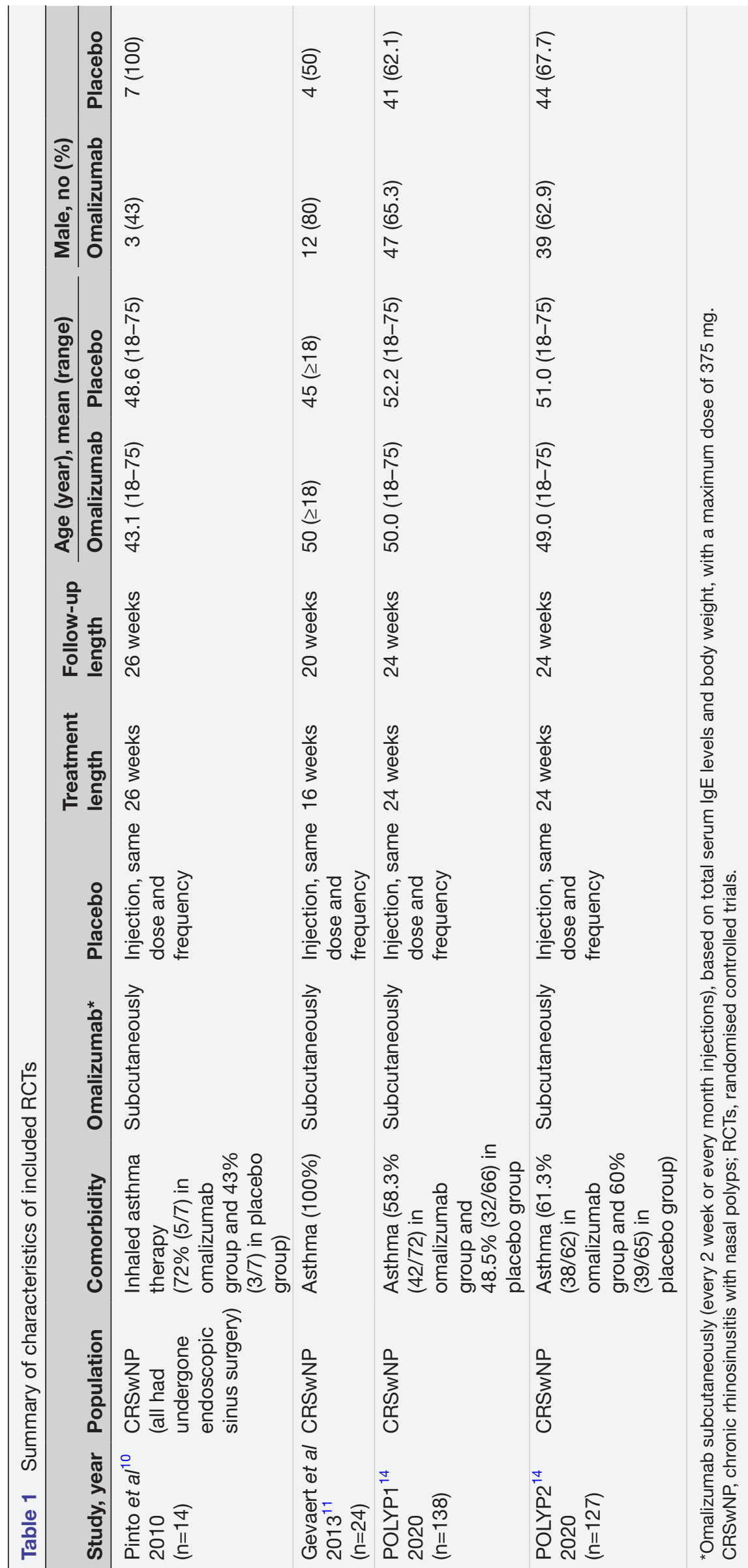




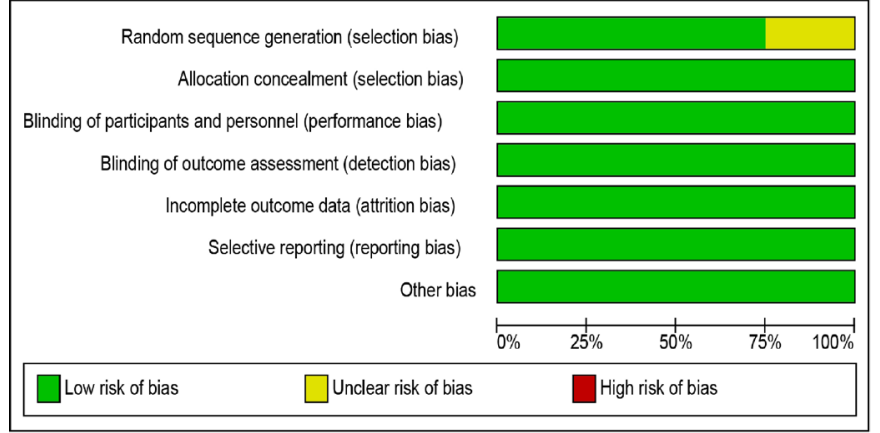

Figure 2 'Risk of bias' graph: review authors' judgements about each risk of bias item presented as percentages across all included studies.

\section{RESULTS}

\section{Study selection}

We identified 1966 studies, of which three (with data for 302 participants) were included in our analysis (figure 1). The three studies (Pinto 2010, ${ }^{10}$ Gevaert $2013^{11}$ and Gevaert $2020^{14}$ were published between 2010 and 2020, of which Gevaert 2020 reported two RCTs (POLYP1 2020 and POLYP2 2020).

\section{Study characteristics}

A summary of key participant characteristics, interventions and comparison pairs was shown in table 1. Except for two participants in Pinto 2010, all the participants were adults with CRSwNP. All the studies were doubleblind RCTs and used a placebo. Study duration ranged from 20 weeks to 26 weeks.

\section{Risk of bias and quality of the clinical trials}

There were four RCTs included in this review. Overall the risk of bias was low, except the random sequence generation of Pinto 2010 was unclear. Our judgements about each risk of bias item presented as percentages across all included studies were shown in figure 2. Our judgements about each risk of bias item for each included study were shown in figure 3 .

\section{Primary outcomes}

Total NPS ranges from 0 to 8 (sum of $0-4$ for left and right nasal passage scores per participant), with a lower score indicating smaller-sized nasal polyps and the highest score indicating large polyps causing complete obstruction of the inferior nasal cavity.

The MD in the change of NPS was -1.20 (95\% CI -1.48 to -0.92 ; 4 RCTs; 302 participants; $\mathrm{I}^{2}=90 \%$; figure $4 \mathrm{~A}$. We noted the high $\mathrm{I}^{2}$ value and Pinto 2020 had no significant reduction in NPS. However, the removal of Pinto 2020 did not change the overall effect size in sensitivity analyses. Therefore, we considered the certainty of the evidence to be high despite the large $\mathrm{I}^{2}$ value.

NCS was assessed daily by the participant via an electronic diary as the response to the following question: Is your nose blocked? The four available response options were scored from 0 (no symptoms) to 3 (severe symptoms).

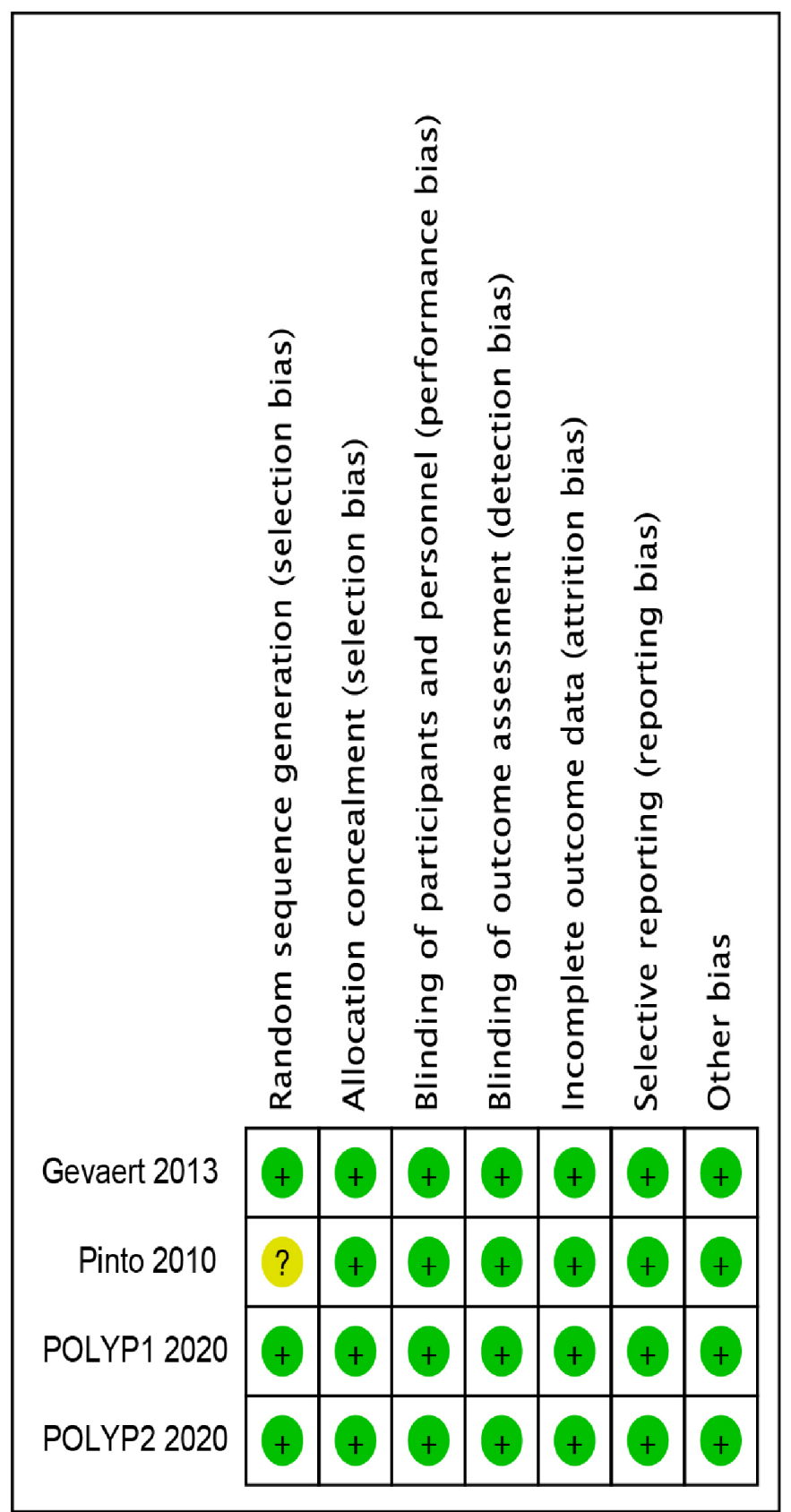

Figure 3 'Risk of bias' summary: review authors' judgements about each risk of bias item for each included study.

The pooled MD of NCS is -0.67 favouring the groups receiving omalizumab (95\% CI -0.86 to -0.48; 3 RCTs; 288 participants; $\mathrm{I}^{2}=82 \%$; figure $\left.4 \mathrm{~B}\right)$. Although the heterogeneity was high in this analysis, all three RCTs showed a significant reduction in NCS with omalizumab.

The MD in the change of SNOT-22 score was 15.62 points lower in participants who received omalizumab (MD=-15.62; 95\% CI -19.79 to $-11.45 ; 265$ participants; $\mathrm{I}^{2}=0 \%$; figure $4 \mathrm{C}$ ). There was an improvement of at least the minimal clinically important difference (MCID; $\geq 8.9$ points).$^{18}$ Because the different measuring tools (Pinto 2010, SNOT-20; Gevaert 2013, Short-Form Health 


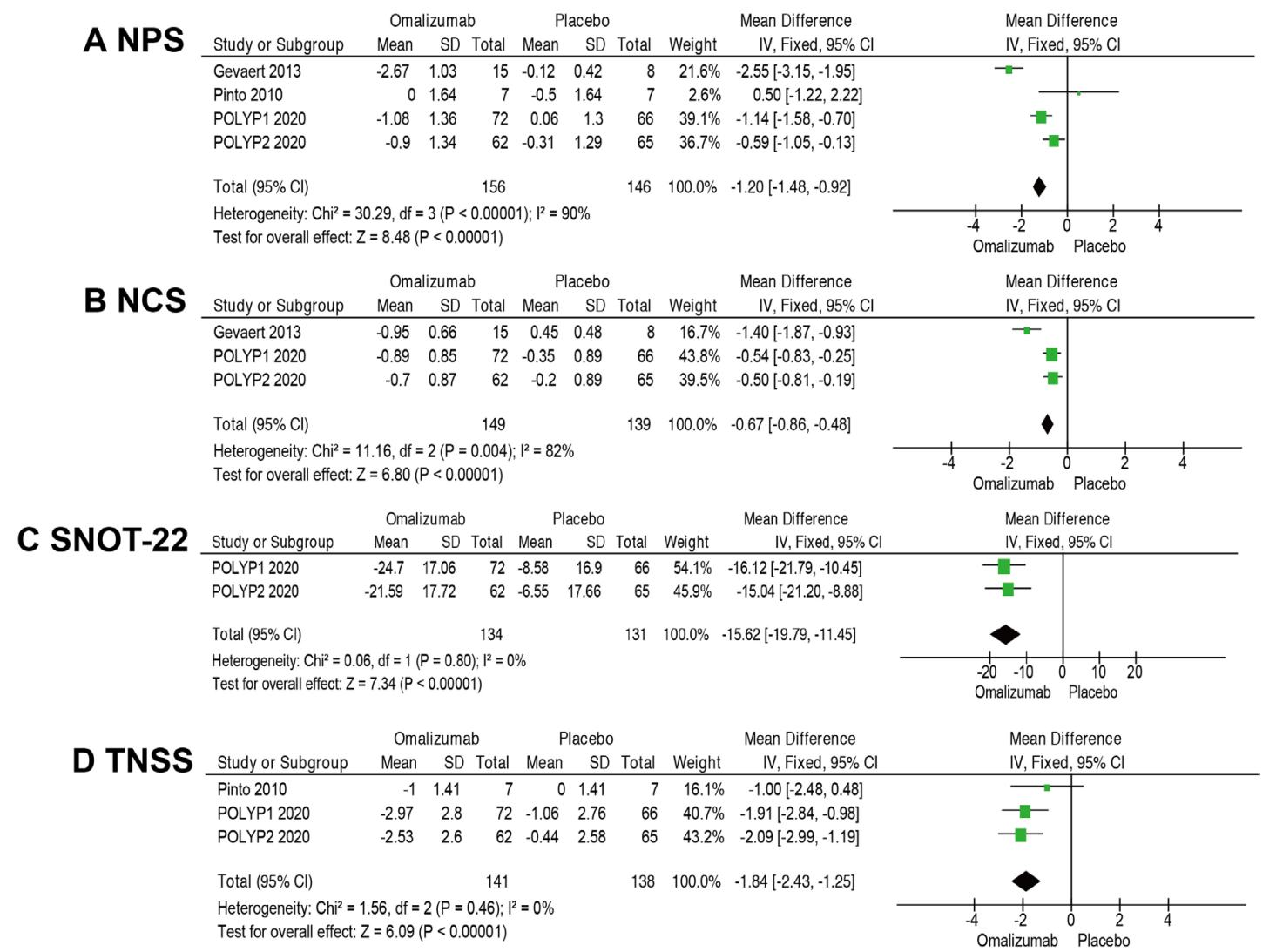

Figure 4 Meta-analyses of omalizumab versus placebo, comparing efficacy and safety. Outcomes assessed are: (A) Nasal Polyps Score (NPS), (B) Nasal Congestion Score (NCS), (C) Sino-Nasal Outcome Test-22 (SNOT-22), and (D) Total Nasal Symptom Score (TNSS).

Questionnaire-36) and unavailable data, these two RCTs were excluded in this pooled analysis.

\section{Secondary outcomes}

TNSS was defined as the sum of the scores for NCS, anterior rhinorrhoea score, posterior rhinorrhoea score and sense of smell score, ranging from 0 (no symptoms) to 12 (most severe symptoms).

The MD in the change of TNSS was 1.84 points lower in omalizumab group ( $\mathrm{MD}=-1.84 ; 95 \% \mathrm{CI}-2.43$ to $-1.25 ; 3$ RCTs; 279 participants; $\mathrm{I}^{2}=0 \%$; figure 4D).

No SAEs were reported in Gevaert 2013 and Pinto 2010. However, POLYP1 2020 reported one case in the placebo group with myocardial infarction and POLYP2 2020 reported one case of pneumonia in the placebo group and three cases in the omalizumab group (one snake bite, one hand fracture and one asthma exacerbation). The pooled result indicated that there was no difference in the risk of SAEs (RR $=1.40 ; 95 \%$ CI 0.29 to 6.80 ; 4 RCTs; 302 participants; $\mathrm{I}^{2}=28 \%$; figure $5 \mathrm{~A}$ ).

There was no difference in the risk of AEs ( $R R=0.83 ; 95 \%$ CI 0.60 to 1.15 ; 4 RCTs; 302 participants; $\mathrm{I}^{2}=0 \%$; figure $5 \mathrm{~B}$ ). It was uncertain where or not there was a difference in the risk of $\mathrm{RSCS}$ (RR=0.52; 95\% CI 0.17 to $1.61 ; 3$ RCTs; 279 participants; $\mathrm{I}^{2}=0 \%$; figure $5 \mathrm{C}$ ).

RNS through week 24 was defined as achievement of NPS of 4 or lower ( $\leq 2$ for each nostril). POLYP1 2020 and POLYP2 2020 reported the number of RNS. The proportion was higher in the group that received omalizumab (RR=5.61; $95 \%$ CI 1.99 to 15.81; 2 RCTs; 265 participants; $\mathrm{I}^{2}=0 \%$; figure $\left.5 \mathrm{D}\right)$.

\section{DISCUSSION}

\section{Principal findings}

This systematic review and meta-analysis identified 4 RCTs with 302 participants evaluating the efficacy and safety of omalizumab in CRSwNP. It showed that omalizumab significantly improved the size of nasal polyps (measured by NPS), symptoms (measured by NCS and TNSS) and (HRQoL; measured by SNOT-22) and reduce the need for surgery (measured by RNS). What is more, there was no difference in the risk of SAEs, AEs and RSCS.

\section{Comparison with other studies}

Hong included two studies (Gavaert 2013 and Pinto 2010) and made a narrative systematic review. ${ }^{12}$ They concluded that there was insufficient evidence to determine the effectiveness of omalizumab for CRS. In Chong's systematic review and meta-analysis, there were three small studies with 65 participants (Gavaert 2013, Pinto 2010 and NCT01066104) evaluated omalizumab. ${ }^{13}$ Their results also showed that there were very uncertain about the effect of omalizumab on disease-specific HRQoL, SAEs, the extent of disease (CT scan scores), generic HRQoL and adverse effects. NCT01066104 ${ }^{19}$ included in Chong's review was 


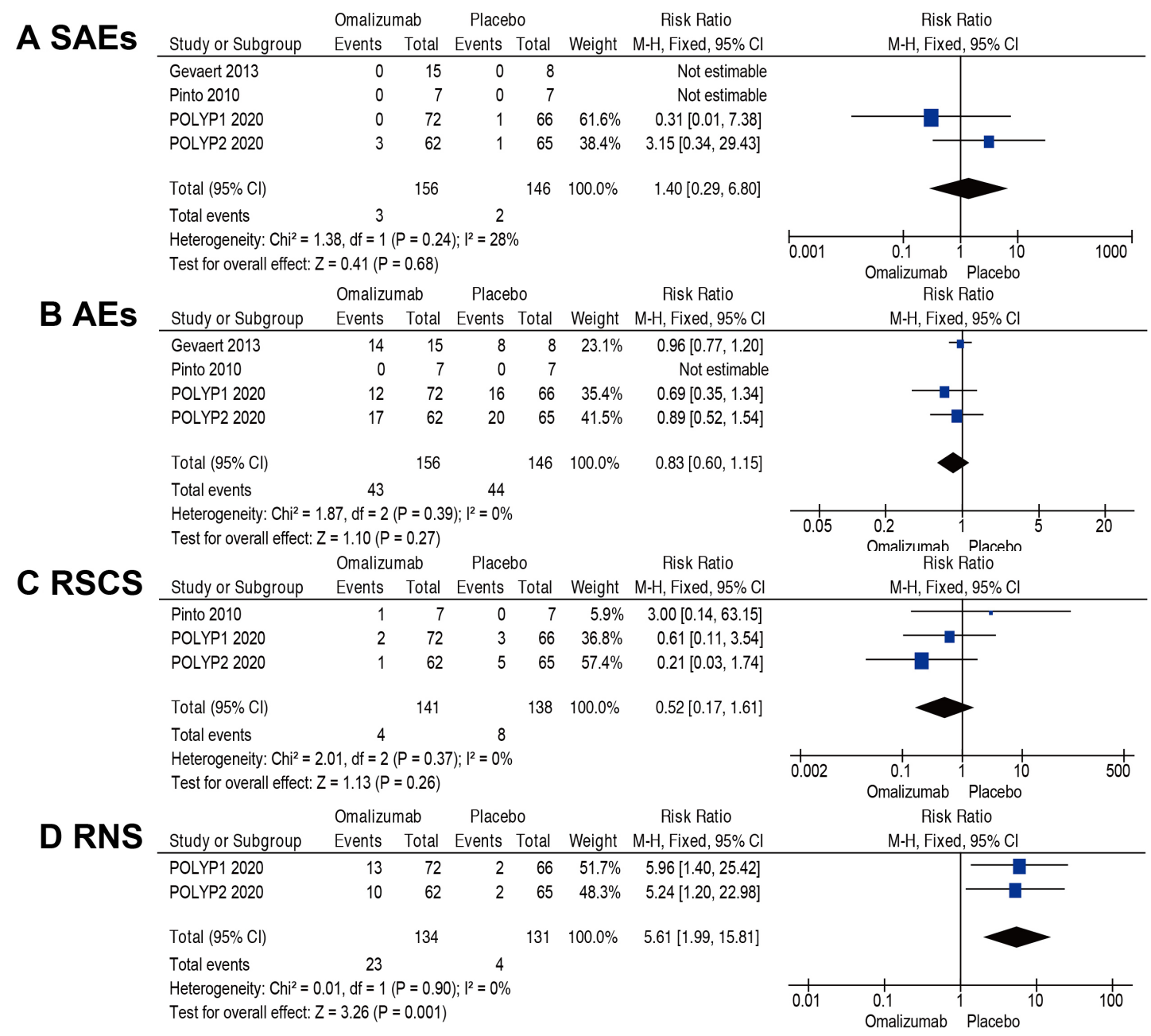

Figure 5 Meta-analyses of omalizumab versus placebo, comparing efficacy and safety. Outcomes assessed are: (A) serious adverse events (SAEs), (B) adverse events (AEs), (C) rescue systemic corticosteroid (RSCS) and (D) reduced need for surgery (RNS). M-H, Mantel-Haenszel.

unpublished data, so it was excluded in our study according to our inclusion criteria.

\section{Implication for future research and clinical practice}

Patients with CRSwNP and comorbid asthma often have a high symptom burden, substantial impact on HRQoL, a higher risk of RSCS and revision surgery. ${ }^{1}$ Moreover, patients with asthma are more likely to develop CRSwNP than are those without asthma, and they are more likely to receive more oral corticosteroid courses. ${ }^{20}$ Therefore, the risk of RSCS may be due to asthma comorbidity.

There were four RCTs included in this systematic review, which recruited patients with moderate to severe CRSwNP. The patients in omalizumab group reduced disease severity and need for surgery, and experienced significant improvements in HRQoL (measured by SNOT-22). Placebocorrected improvements of SNOT-22 was 15.6 points, which exceeded the commonly accepted MCID of 8.9 points. ${ }^{18} 21$ Furthermore, there was no increased risk of SAEs and AEs in patients treated with omalizumab. Thus, it was certain that omalizumab significantly improved endoscopic, clinical, and patient-reported outcomes in moderate to severe CRSwNP and it was well tolerated.
However, it is still unknown that omalizumab is effective in patients with less severe CRSwNP (such as NPS=1 for each nostril or unilateral nostril) and more affordable compared with conventional treatment with topical and SCS and surgery. Therefore, studies are required to evaluate their effectiveness in patients with less severe diseases and their cost in the treatment. In addition, long-term observational studies are also required to determine if omalizumab lose its effectiveness over time, or whether there are any late AEs.

\section{Limitations of the study}

Despite the strict methodology of this systematic review and meta-analysis using PRISMA guidelines, certain limitations should be considered. First, four RCTs were recruited from the same group with moderate to severe CRSwNP. Therefore, there is no evidence on whether or not patients with less severe CRSwNP (NPS=1 for each nostril or unilateral nostril) would benefit. Second, four RCTs were all in adults and no available data for children. Third, because the longest follow-up of four RCTs was only up to 26 weeks, there were too short to comprehensively and adequately assess the risks of side effect, RSCS and RNS. Finally, there were only four RCTs $(<10)$, so a possibility of publication 
bias was not assessed by constructing a funnel plot in this systematic review. ${ }^{17}$

\section{CONCLUSIONS}

To the best of our knowledge, this was the first meta-analysis that identified omalizumab significantly improved endoscopic, clinical and patient-reported outcomes in moderate to severe CRSwNP and it was safe and well tolerated. Studies are required to evaluate their effectiveness in patients with less severe diseases and their cost in the treatment.

\section{Author affiliations}

${ }^{1}$ Department of Otorhinolaryngology-Head and Neck Surgery, The Third Affiliated Hospital of Sun Yat-Sen University, Guangzhou, People's Republic of China ${ }^{2}$ Department of Allergy, The Third Affiliated Hospital of Sun Yat-Sen University, Guangzhou, People's Republic of China

${ }^{3}$ Department of Science and Research, The Third Affiliated Hospital of Sun Yat-Sen University, Guangzhou, People's Republic of China

Contributors Concept and design: RZ and QY. Acquisition, analysis or interpretation of data: $\mathrm{QW}, \mathrm{LY}, \mathrm{HQ}, \mathrm{XW}$ and $\mathrm{X}-\mathrm{KH}$. Drafting of the manuscript: $\mathrm{QW}$ and $\mathrm{LY}$. Critical revision of the manuscript for important intellectual content: $R Z$ and $Q Y$. Statistical analysis: LY. Supervision: RZ and QY.

Funding This work was supported by the National Natural Science Foundation of China (No. U20A20399 and 81870704), the Key-area Research and Development Programme of Guangdong Province (No.2020B0101130015), Sun Yat-sen University Clinical Research 5010 Program (No. 2019006), the China Postdoctoral Science Foundation Grant (2019M653207), Guangdong Basic and Applied Basic Research Foundation (2020A1515010133) and the Medical Scientific Research Foundation of Guangdong Province of China (A2019037).

Competing interests None declared.

Patient consent for publication Not required.

Provenance and peer review Not commissioned; externally peer reviewed.

Data availability statement Data are available in a public, open access repository. All data relevant to the study are included in the article or uploaded as supplementary information.

Supplemental material This content has been supplied by the author(s). It has not been vetted by BMJ Publishing Group Limited (BMJ) and may not have been peer-reviewed. Any opinions or recommendations discussed are solely those of the author(s) and are not endorsed by BMJ. BMJ disclaims all liability and responsibility arising from any reliance placed on the content. Where the content includes any translated material, BMJ does not warrant the accuracy and reliability of the translations (including but not limited to local regulations, clinical guidelines, terminology, drug names and drug dosages), and is not responsible for any error and/or omissions arising from translation and adaptation or otherwise.

Open access This is an open access article distributed in accordance with the Creative Commons Attribution Non Commercial (CC BY-NC 4.0) license, which permits others to distribute, remix, adapt, build upon this work non-commercially, and license their derivative works on different terms, provided the original work is properly cited, appropriate credit is given, any changes made indicated, and the use is non-commercial. See: http://creativecommons.org/licenses/by-nc/4.0/.
ORCID iD

Qintai Yang http://orcid.org/0000-0003-3377-737X

\section{REFERENCES}

1 Fokkens WJ, Lund VJ, Hopkins C, et al. European position paper on rhinosinusitis and nasal polyps 2020. Rhinology 2020;58:1-464.

2 Benjamin MR, Stevens WW, Li N, et al. Clinical characteristics of patients with chronic rhinosinusitis without nasal polyps in an academic setting. J Allergy Clin Immunol Pract 2019;7:1010-6.

3 Bachert C, Zhang L, Gevaert P. Current and future treatment options for adult chronic rhinosinusitis: focus on nasal polyposis. J Allergy Clin Immunol 2015;136:1431-40.

4 Khan A, Huynh TMT, Vandeplas G, et al. The Galen rhinosinusitis cohort: chronic rhinosinusitis with nasal polyps affects health-related quality of life. Rhinology 2019;57:343-51.

5 Rudmik L. Economics of chronic rhinosinusitis. Curr Allergy Asthma Rep 2017;17:20.

6 Stevens WW, Schleimer RP, Kern RC. Chronic rhinosinusitis with nasal polyps. J Allergy Clin Immunol Pract 2016;4:565-72.

7 Tokunaga T, Sakashita M, Haruna T, et al. Novel scoring system and algorithm for classifying chronic rhinosinusitis: the JESREC study. Allergy 2015;70:995-1003.

8 DeConde AS, Mace JC, Levy JM, et al. Prevalence of polyp recurrence after endoscopic sinus surgery for chronic rhinosinusitis with nasal polyposis. Laryngoscope 2017;127:550-5.

9 Calus L, Van Bruaene N, Bosteels C, et al. Twelve-year followup study after endoscopic sinus surgery in patients with chronic rhinosinusitis with nasal polyposis. Clin Transl Allergy 2019;9:30.

10 Pinto JM, Mehta N, DiTineo M, et al. A randomized, double-blind, placebo-controlled trial of anti-lgE for chronic rhinosinusitis. Rhinology 2010;48:318-24.

11 Gevaert P, Calus L, Van Zele T, et al. Omalizumab is effective in allergic and nonallergic patients with nasal polyps and asthma. $J$ Allergy Clin Immunol 2013;131:e111. 2012/10/02:110-6.

12 Hong CJ, Tsang AC, Quinn JG, et al. Anti-IgE monoclonal antibody therapy for the treatment of chronic rhinosinusitis: a systematic review. Syst Rev 2015;4:166.

13 Chong L-Y, Piromchai P, Sharp S, et al. Biologics for chronic rhinosinusitis. Cochrane Database Syst Rev 2020;2:CD013513.

14 Gevaert P, Omachi TA, Corren J, et al. Efficacy and safety of omalizumab in nasal polyposis: 2 randomized phase 3 trials. $J$ Allergy Clin Immunol 2020;146:595-605.

15 Wu Q, Zheng R, Qiu H. Anti-IgE monoclonal antibody in the treatment of chronic rhinosinusitis with nasal polyps. Prospero 2020 CRD42020207639. Available: https://www.crd.york.ac.uk/prospero/ display_record.php?ID=CRD42020207639

16 Moher D, Liberati A, Tetzlaff J, et al. Preferred reporting items for systematic reviews and meta-analyses: the PRISMA statement. Ann Intern Med 2009;151:264.

17 Higgins JPT, Green S, eds. Cochrane handbook for systematic reviews of interventions version 5.1.0. The Cochrane Collaboration, 2011. www.cochrane-handbook.org

18 Chowdhury NI, Mace JC, Bodner TE, et al. Investigating the minimal clinically important difference for SNOT-22 symptom domains in surgically managed chronic rhinosinusitis. Int Forum Allergy Rhinol 2017;7:1149-55.

19 NCT01066104. subcutaneous omalizumab for treatment of chronic rhinosinusitis with nasal polyposis, 2009. Available: https:// clinicaltrials.gov/show/nct01066104

20 John Staniorski C, Price CPE, Weibman AR, et al. Asthma onset pattern and patient outcomes in a chronic rhinosinusitis population. Int Forum Allergy Rhinol 2018;8:495-503.

21 Hopkins C, Gillett S, Slack R, et al. Psychometric validity of the 22item sinonasal outcome test. Clin Otolaryngol 2009;34:447-54. 